\title{
Role of methylene blue in detecting the sentinel lymph node in colorectal cancer: In vivo vs. ex vivo technique
}

\author{
DANIEL STANILOAIE ${ }^{1,2^{*}}$, CONSTANTIN BUDIN $^{1 *}$, DANUT VASILE $^{1,2}$, GEORGE IANCU $^{3}$, ALEXANDRU ILCO $^{2}$, \\ DANIEL IULIAN VOICULESCU ${ }^{1,2}$, ALEXANDRA FLORINA TRANDAFIR ${ }^{1,2}$, TAREK AMMAR $^{2}$, EMEL SULIMAN $^{1,2}$, \\ EMINE SULIMAN ${ }^{4}$, DORIN DRAGOȘ,6 and MARIA-DANIELA TANASESCU ${ }^{5,7 *}$
}

\begin{abstract}
${ }^{1}$ Department of General Surgery, Faculty of Medicine, 'Carol Davila' University of Medicine and Pharmacy, 020021 Bucharest; ${ }^{2}$ st Department of General Surgery, Bucharest Emergency University Hospital, 050098 Bucharest;

${ }^{3}$ Discipline of Obstetrics and Gynecology, Filantropia Clinical Hospital Faculty of Medicine, 'Carol Davila' University of Medicine and Pharmacy, 011171 Bucharest; ${ }^{4}$ Department 3 - Complementary Sciences, Discipline of Medical Informatics and Biostatistics, Faculty of Medicine, 'Carol Davila' University of Medicine and Pharmacy;

${ }^{5}$ Department of Medical Semiology, Discipline of Internal Medicine I and Nephrology, Faculty of Medicine,

'Carol Davila' University of Medicine and Pharmacy, 020021 Bucharest; ${ }^{6} 1$ st Department of Internal Medicine;

${ }^{7}$ Department of Nephrology, Emergency University Hospital, 050098 Bucharest, Romania
\end{abstract}

Received September 1, 2021; Accepted October 1, 2021

DOI: $10.3892 / \mathrm{etm} .2021 .10995$

\begin{abstract}
The identification of sentinel lymph nodes is a valuable oncological method, which aims at mapping lymphatic drainage and has the advantage of correctly staging the disease and assessing prognosis. Lymph node invasion is an important prognostic feature. In colorectal cancer, lymphadenectomy is not influenced by the positive or negative status of the sentinel lymph node. The identification of lymph nodes with possible invasion by staining the primary tumor with methylene blue can lead to improved staging and management. In other words, the consequent administration of neoadjuvant therapy (chemotherapy) to the appropriate patients may result in lower recurrence rates. Thus, the aim of the present study was to use methylene blue to identify the sentinel node/nodes in colorectal cancer and to determine whether the dye-capturing nodes were invaded by the tumor. This is a non-randomized prospective study, in which 26 patients with colon cancer with surgical indication were enrolled. Two types of methods were utilized: in vivo (16 patients) and ex vivo (10 patients). The identification rate was $75 \%$ for the in vivo technique and $60 \%$ for the ex vivo technique, resulting in a $69.26 \%$ overall identification rate. Of 18 patients with sentinel lymph nodes
\end{abstract}

Correspondence to: Dr Constantin Budin, Department of General Surgery, Faculty of Medicine, 'Carol Davila' University of Medicine and Pharmacy, 37 Dionisie Lupu Street, 020021 Bucharest, Romania E-mail: constantin.budin@umfcd.ro

*Contributed equally

Key words: sentinel lymph node, lymphadenectomy, methylene blue, colorectal cancer, oncology identified using dye, routine histological examination detected metastases in $6(33.33 \%)$ of these patients. In conclusion, further research should be conducted into how the clinical application of sentinel node detection can be employed in colorectal cancer.

\section{Introduction}

Colorectal cancer is the third most commonly diagnosed malignancy worldwide, with approximately 1.9 million new cases diagnosed in 2020, for both sexes (1). Lymph node status is the best predictive marker for recurrence and survival in patients with colorectal cancer (2). The survival rates are dependent on TNM stage: in Stage I and II the 5-year survival rates are between 82 and $93 \%$, decreasing to $59 \%$ in the presence of lymph node metastases (Stage III) (3). The main prognostic factor in colorectal cancer is the lymph node stage. The 5-year survival rate is approximately $80 \%$ in patients with disease-free lymph nodes but is reduced to $50 \%$ in patients with one metastatic lymph node (4).

Ernest A. Gould introduced for the first time in 1960 the sentinel lymph node term, while performing a total parotidectomy for a mixed tumor. The lymph node was sent to the pathologist and the report was 'lymph node with metastatic tumor' (5). Ramon M. Cabanas identified in 1977 the sentinel lymph node of a penile carcinoma (6). For colon cancer, the term was introduced in 1999 by Joosten et al in a study on 50 patients (7). However, the study showed a high percentage of false-negative results.

Lymph nodes that drain directly from the tumor area are known as sentinel nodes and are believed to be the first site for metastasis (8).

Positive lymph nodes can be missed when an insufficient number of lymph nodes are sampled. Although severely enlarged lymph nodes often contain a tumor, especially when 
they have lost their contour, lymph nodes harboring metastases are not necessarily enlarged (9).

The sentinel lymph node concept is based on the premise that lymphatic drainage of tumors initially passes through a single or small number of lymph nodes prior to spreading to more distant lymph nodes (10). The sentinel lymph node is therefore the lymph node most likely to harbor metastatic cells (11). A review of the subject suggested that an 'optimal number of lymph nodes' is unlikely to exist and depends on a variety of factors (12).

In melanoma and breast cancer, determining the status of the sentinel node can prevent unnecessary lymph node dissection and the associated high morbidity. In colorectal cancer, knowing the status (positive or negative) of the sentinel node does not influence the surgical technique of lymph node dissection as this must be performed completely regardless. However, it may improve the detection of occult disease harboring lymph nodes, leading to increased diagnostic accuracy (13).

In the present study, methylene blue was injected peritumorally, both in vivo and ex vivo, with the aim of identifying the sentinel lymph nodes and mapping the lymphatic drainage of the tumor. Methylene blue is a monovalent cationic dye with a molecular formula of $\mathrm{C}_{16} \mathrm{H}_{18} \mathrm{C}_{1} \mathrm{~N}_{3} \mathrm{~S}$ (14).

\section{Patients and methods}

Patient characteristics. This is a prospective non-randomized study, in which 26 patients (14 female, 12 male) were enrolled, with ages ranging from 35 to 83 years and clustering in the 60-79 age interval. The patients were admitted to the $1 \mathrm{st}$ General Surgery Department of the University Emergency Hospital, Bucharest (Romania) between January 2018 and February 2020. The present study was approved by the Ethics Committee of the University Emergency Hospital, Bucharest. Written informed consent was obtained from the patients prior to publication.

Inclusion and exclusion criteria. The inclusion criterion was the existence of a colorectal cancer amenable to curative surgery.

The exclusion criteria were: i) the coexistence of another malignant tumor (synchronous tumor); ii) the presence of metastatic tumors; iii) pregnancy or breastfeeding; iv) preoperative chemotherapy. Methylene blue was used to identify lymph nodes. Two techniques, in vivo and ex vivo, were employed to identify the sentinel node and the lymphatic territory.

Method. The aim of the present study was to determine whether the sentinel node detection technique, already confirmed and standardized in the surgical treatment of breast cancer and melanoma (15), is also applicable to colorectal cancer.

For an improved description, the demographic background was also taken into account, revealing a preponderance of urban (18 patients) over rural (8 patients) background.

Preoperative evaluation included a complete history, physical examination, complete blood count, tumor markers (carcinoembryonic antigen, CA 19-9), serum calcium, total proteins, albumin. Patients were examined by colonoscopy with biopsy; the malignancy was confirmed by histopathological examination, and thereafter a CT scan was performed.
The sentinel node identification relied on injecting $1 \%$ methylene blue either in vivo (in 16 cases) or ex vivo (in 10 cases). Written informed consent was obtained from each patient prior to the intravital dye injection. The study was conducted after being approved by the local bioethics committee.

The surgical techniques employed were right hemicolectomy (13 cases), extended right hemicolectomy ( 1 case), left hemicolectomy ( 2 cases), Dixon (3 cases), and Hartmann (7 cases).

Technique for in vivo identification of sentinel node and lymphatic mapping. The intervention started with exploratory laparotomy to confirm the site of the primary tumor, to rule out any distant metastases, and to ensure that no other exclusion criteria are met.

A total volume of $2 \mathrm{ml}$ of $1 \%(10 \mathrm{mg} / \mathrm{ml})$ methylene blue was injected subserosally, $0.5 \mathrm{ml}$ at each cardinal point, using a 26 Gauge needle syringe. In order to reduce the risk of spreading tumor fragments into the lymphatic system, no massage was performed on the tumor after dye injection.

After a time-lapse of 1-10 min following the injection, the identification of the first lymph nodes became possible by inspecting the mesocolon (16). The lymphadenectomy area was established so as to include all the stained lymph nodes; thereafter the corresponding vascular pedicles were ligated.

After the complete excision of the tumor and the lymph nodes, the sentinel node was separately sent to the Department of Pathological Anatomy. The location of the tumor was cecum (9 cases), ascending colon (4 cases), right flexure (1 case), descending colon (1 case), sigmoid (5 cases), rectosigmoid junction ( 3 cases), and rectum ( 3 cases). In none of the cases was the tumor situated in the transverse colon or in the left flexure.

Technique for ex vivo identification of sentinel node and lymphatic mapping. This technique consists of injecting $1 \%$ $(10 \mathrm{mg} / \mathrm{ml})$ methylene blue, $0.25-1 \mathrm{ml}$ in each cardinal point, 15-30 min after removing the excision section from the peritoneal cavity (17) and cutting it open on the antimesenteric area (18). The volume of injected dye varies considerably (from 0.25 to $5.0 \mathrm{ml}$ ) in previously published reports (19-26).

In the present study, the dye was injected subserosally in a manner similar to that used in the in vivo technique: $0.5 \mathrm{ml}$ at each cardinal point (amounting to a total of $2 \mathrm{ml}$ ), using a 26 Gauge needle syringe.

The sentinel node/s that captured the dye in the first 5-10 min after injection (10) were marked by suture threads and then excised and sent separately to the Department of Pathological Anatomy.

Previous findings suggested that the dye may be injected submucosally during colonoscopy, with the drawbacks of elevating the risk for the patient, of being time consuming, and of lacking a scientifically proven benefit over subserosal injection during open colon surgery (27).

It seems that cardiovascular disease, diabetes, diabetic arteriopathy, and the severity of arterial wall calcification are relevant for the amount of blood perfusion to the colon, as well as for the dye uptake by the lymph nodes $(28,29)$. To the best of our knowledge, no studies have investigated a putative relationship between hypocalcemia and the degree of lymph node invasion in colorectal cancer. 
Table I. Characteristics of the study group.

\begin{tabular}{|c|c|c|c|c|c|c|c|c|c|c|}
\hline \multirow[b]{2}{*}{$\begin{array}{l}\text { Case } \\
\text { no. }\end{array}$} & \multirow[b]{2}{*}{ Location } & \multirow[b]{2}{*}{ pT } & \multirow[b]{2}{*}{$\mathrm{pN}$} & \multirow[b]{2}{*}{ G } & \multirow[b]{2}{*}{ Stage } & \multirow{2}{*}{$\begin{array}{l}\text { Type of } \\
\text { identification } \\
\text { technique }\end{array}$} & \multicolumn{4}{|c|}{ No. of lymph nodes } \\
\hline & & & & & & & Examined & Invaded & Sentinel & $\begin{array}{l}\text { Sentinel } \\
\text { invaded }\end{array}$ \\
\hline 1 & Recto-sigmoid & pT3 & $\mathrm{pN} 2 \mathrm{~b}$ & G2 & IIIC & Ex vivo & 26 & 10 & 3 & 2 \\
\hline 2 & Sigmoid & pT3 & pNO & G2 & IIA & In vivo & 11 & 0 & 0 & 0 \\
\hline 3 & Sigmoid & pT3 & pNo & $\mathrm{G} 2$ & IIA & In vivo & 25 & 0 & 1 & 0 \\
\hline 4 & Cecum & pT3 & pN0 & $\mathrm{G} 2$ & IIA & In vivo & 17 & 0 & 0 & 0 \\
\hline 5 & Cecum & pT3 & $\mathrm{pN} 2 \mathrm{a}$ & $\mathrm{G} 2$ & IIIB & In vivo & 26 & 4 & 2 & 1 \\
\hline 6 & Ascending & pT3 & pNO & G3 & IIA & In vivo & 26 & 0 & 1 & 0 \\
\hline 7 & Recto-sigmoid & pT3 & $\mathrm{pN} 2 \mathrm{~b}$ & G3 & IIIC & Ex vivo & 23 & 7 & 2 & 2 \\
\hline 8 & Recto-sigmoid & pT3 & pNO & G2 & IIA & Exvivo & 18 & 0 & 1 & 0 \\
\hline 9 & Ascending & pT2 & pNo & G2 & IIA & In vivo & 12 & 0 & 1 & 0 \\
\hline 10 & Cecum & pT3 & pNO & G3 & IIA & In vivo & 9 & 0 & 1 & 0 \\
\hline 11 & Sigmoid & pT4a & $\mathrm{pN} 2 \mathrm{~b}$ & $\mathrm{G} 2$ & IIIC & In vivo & 14 & 14 & 3 & 2 \\
\hline 12 & Sigmoid & pT3 & $\mathrm{pN} 1 \mathrm{~b}$ & $\mathrm{G} 2$ & IIIB & In vivo & 16 & 3 & 1 & 1 \\
\hline 13 & Recto-sigmoid & pT4a & pNO & $\mathrm{G} 2$ & IIB & Ex vivo & 32 & 0 & 0 & 0 \\
\hline 14 & Sigmoid & pT3 & $\mathrm{pN} 1$ & $\mathrm{G} 2$ & IIIB & Ex vivo & 10 & 1 & 1 & 0 \\
\hline 15 & Cecum & pT4a & $\mathrm{pN} 1 \mathrm{c}$ & G1 & IIIB & In vivo & 16 & 0 & 1 & 0 \\
\hline 16 & Ascending & pT4a & pNO & $\mathrm{G} 2$ & IIB & Ex vivo & 6 & 0 & 1 & 0 \\
\hline 17 & Descending & pT3 & pN1b & $\mathrm{G} 2$ & IIIB & In vivo & 10 & 3 & 1 & 1 \\
\hline 18 & Cecum & pT3 & pNO & G1 & IIA & In vivo & 10 & 0 & 1 & 0 \\
\hline 19 & Cecum & pT2 & pNO & $\mathrm{G} 2$ & I & Exvivo & 17 & 0 & 1 & 0 \\
\hline 20 & Right hepatic flexure & pT4 & pN1c & $\mathrm{G} 2$ & IIIB & Ex vivo & 23 & 0 & 0 & 0 \\
\hline 21 & Cecum & pT2 & pNO & $\mathrm{G} 2$ & I & In vivo & 21 & 0 & 1 & 0 \\
\hline 22 & Middle rectum & pT3 & pN0 & G2 & IIA & Ex vivo & 10 & 0 & 0 & 0 \\
\hline 23 & Cecum & pT2 & pNo & $\mathrm{G} 2$ & I & In vivo & 21 & 0 & 1 & 0 \\
\hline 24 & Cecum & pT3 & pNo & G3 & II & In vivo & 33 & 0 & 0 & 0 \\
\hline 25 & Middle rectum & pT1 & pNo & G1 & I & In vivo & 2 & 0 & 0 & 0 \\
\hline 26 & Ascending & pT3 & pNO & $\mathrm{G} 2$ & IIA & Ex vivo & 23 & 0 & 0 & 0 \\
\hline
\end{tabular}

For tumor staging and case classification, we used the TNM system of the American Joint Committee on Cancer (AJCC) (30).

Table I presents the characteristics of the study group, tumor histologic grade, lymph nodes examined and TNM staging.

Statistical analysis. Fisher's exact test was employed to determine the statistical significance of the results. All the statistical computations were performed using the $\mathrm{R}$ language and environment for statistical computing and graphics version 4.0.3 (R Foundation for Statistical Computing Platform).

\section{Results and Discussion}

The in vivo technique is preferred because it has the advantage of identifying aberrant lymphatic drainage that can guide lymphadenectomy $(31,32)$. In 18 cases, the sentinel node was macroscopically identified on the resected section: 12 in vivo and 6 ex vivo. In 8 cases, no lymph nodes were stained (Table I).

In order to attain a high level of accuracy, 10-12 lymph nodes should be examined $(33,34)$.
A study performed on 45 patients diagnosed with colon cancer used a combination of methylene blue and technetium $(99 \mathrm{mTc})$. A few minutes after injecting the tracers, the colon and its mesentery were visually examined for any blue-stained lymph nodes and a hand-held gamma probe was employed for detecting areas of high radioactivity. Sentinel nodes were successfully identified in 43 patients (95.6\%) with a mean of 1.7 nodes/patient (2).

Of the 18 positive cases in the present study, only 1 sentinel node was identified in the majority, i.e., 14 of the 18 cases, 2 sentinel nodes in 2 cases, and 3 sentinel nodes in the remaining 2 cases (Table I).

The overall identification rate was $69.26 \%$ (18/26 cases). The identification rate was $75 \%$ for the in vivo technique (12/16 cases) and $60 \%$ for the ex vivo technique (6/10 cases). Although the difference was not statistically significant $(\mathrm{P}=0.6645$, Fisher's exact test) a higher identification rate was expected owing to the in vivo technique because it has the advantage of preserved lymph flow as it is performed before the ligation of the main vascular pedicles, while the ex vivo technique is inherently plagued by a partially compromised lymph flow, as it is performed after removing the excision section. 
Methylene blue-stained lymph nodes were microscopically examined after hematoxylin and eosin staining. Of the 18 patients with dye-identified sentinel nodes, routine histological examination demonstrated metastases in 6 cases $(33.33 \%)$, of which 3 cases had one tumor-ridden sentinel node, 2 had 2 tumor-burdened out of 3 sentinel nodes, and 1 had 1 invaded out of 2 sentinel nodes (Table I).

Complete lymphadenectomy was performed in all 26 cases, regardless of the staining status of the lymph nodes.

The cases in which the lymph nodes were free of metastasis required further evaluation in search for micrometastases (skip metastases) using immunohistochemistry or reverse transcription polymerase chain reaction (RT-PCR) techniques, but these methods are much more expensive, labor-intensive and/or time-consuming.

Sentinel lymph node biopsy in colorectal cancer is an extremely controversial issue. The sentinel node identification by means of $1 \%$ methylene blue dying is a feasible, costeffective method.

The learning curve is particularly important for increasing the accuracy of the technique and is responsible for the negative results in some cases. To increase the rate of sentinel lymph node/nodes identification by means of methylene blue staining, a longer learning curve is needed.

For the time being, the clinical application of sentinel node detection used in breast cancer and melanoma cannot be successfully transferred to colon cancer. Further studies are needed to determine the specificity of the technique in this pathology.

\section{Acknowledgements}

Not applicable.

\section{Funding}

No funding was received.

\section{Availability of data and materials}

All data generated or analyzed during this study are included in this published article.

\section{Authors' contributions}

DS, CB, DV, GI, AI, DIV, AFT, TA EMES, EMIS, DD, and MDT designed the study, wrote the manuscript and performed the literature research. DV and AI evaluated and are responsible for confirming authenticity of all the raw data, critically revised the manuscript and analyzed the results for accuracy. All authors read and approved the final manuscript. The contributions of all the authors to this study are greatly valued and appreciated.

\section{Ethics approval and consent to participate}

This study was approved by Ethics Committee of Bucharest Emergency University Hospital, Romania with the approval no. 55321. Written informed consent was obtained from the patients prior to publication.

\section{Patient consent for publication}

Not applicable.

\section{Competing interests}

The authors declare that they have no competing interests.

\section{References}

1. Sung H, Ferlay J, Siegel RL, Laversanne M, Soerjomataram I, Jemal A and Bray F: Global cancer statistics 2020. GLOBOCAN estimates of incidence and mortality worldwide for 36 cancers in 185 countries. CA Cancer J Clin 71: 209-249, 2021.

2. Hokkam E, El-Kammash S, Abdelaziz A, Farrag S, Fathy H and Gomaa A: Assessment of sentinel lymph node biopsy in colon cancer and its impact on staging. Journal of Surgery 4: 36-40, 2016.

3. O'Connell JB, Maggard MA and Ko CY: Colon cancer survival rates with the new American joint committee on cancer sixth edition staging. J Natl Cancer Inst 96: 1420-1425, 2004.

4. Carrara A, Motter M, Amabile D, Pellecchia L, Moscatelli P, Pertile R, Barbareschi M, Decarli NL, Ferrari M and Tirone G: Predictive value of the sentinel lymph node procedure in the staging of non-metastatic colorectal cancer. Int J Colorectal Dis 35: 1921-1928, 2020.

5. Gould E, Winship T, Philbin P and Kerr H: Observations on a 'sentinel node' in cancer of the parotid. Cancer 13: 77-78, 1960.

6. Cabanas RM: An approach for the treatment of penile carcinoma. Cancer 39: 456-466, 1977.

7. Joosten JJ, Strobbe LJ, Wauters CA, Pruszczynski M, Wobbes T and Ruers TJ: Intraoperative lymphatic mapping and the sentinel node concept in colorectal carcinoma. Br J Surg 86: 482-486, 1999.

8. Andersen HS, Bennedsen ALB, Burgdorf SK, Eriksen JR, Eiholm S, Toxværd A, Riis LB, Rosenberg J and Gögenur I: In vivo and ex vivo sentinel node mapping does not identify the same lymph nodes in colon cancer. Int J Colorectal Dis 32: 983-990, 2017.

9. Braat AE, Oosterhuis JW, de Vries JE and Tollenaar RA: Lymphatic staging in colorectal cancer: Pathologic, molecular, and sentinel node techniques. Dis Colon Rectum 48: 371-383, 2005.

10. Fitzgerald TL, Khalifa MA, Al Zahrani M, Law CH and Smith AJ: Ex vivo sentinel lymph node biopsy in colorectal cancer: A feasibility study. J Surg Oncol 80: 27-32, 2002.

11. Albayrak Y, Oren D, Gündoğdu C and Kurt A: Intraoperative sentinel lymph node mapping in patients with colon cancer: Study of 38 cases. Turk J Gastroenterol 22: 286-292, 2011.

12. Minhas JS: Lymph node correlations and thresholds in colorectal cancer specimens. Gastrointest Cancer Res 5 (3 Suppl 1): S31, 2012.

13. Huynh KT and Bilchik AJ: Sentinel lymph node biopsy and nodal ultrastaging in colorectal cancer. Cancer J 21: 11-16, 2015.

14. Elmorsi T: Equilibrium isotherms and kinetic studies of removal of methylene blue dye by adsorption on to miswak leaves as a natural adsorbent. J Environmental Protection 5: 817-827, 2011.

15. Cahill RA: What's wrong with sentinel node mapping in colon cancer? World J Gastroenterol 13: 6291-6294, 2007.

16. Mulsow J, Winter DC, O'Keane JC and O'Connell PR: Sentinel lymph node mapping in colorectal cancer. Br J Surg 90: 659-667, 2003.

17. Van Schaik PM, van der Linden JC, Ernst MF, Gelderman WA and Bosscha K: Ex vivo sentinel lymph node 'mapping' in colorectal cancer. Eur J Surg Oncol 33: 1177-1182, 2007

18. Wong JH, Steineman S, Calderia C, Bowles J and Namiki T: Ex vivo sentinel node mapping in carcinoma of the colon and rectum. Ann Surg 233: 515-521, 2001.

19. Stojadinovic A, Allen PJ, Protic M, Potter JF, Shriver CD, Nelson JM and Peoples GE: Colon sentinel lymph node mapping: practical surgical applications. J Am Coll Surg 201: 297-313, 2005.

20. Chan $\mathrm{SH}, \mathrm{Ng} \mathrm{C}$ and Looi LM: Intraoperative methylene blue sentinel lymph node mapping in colorectal cancer. ANZ J Surg 9: 775-779, 2008. 
21. Märkl B, Schaller T, Krammer I, Cacchi C, Arnholdt HM, Schenkirsch G, Kretsinger H, Anthuber M and Spatz H: Methylene blue-assisted lymph node dissection technique is not associated with an increased detection of lymph node metastases in colorectal cancer. Mod Pathol 9: 1246-1254, 2013.

22. Ceranic MS, Kecmanovic DM, Pavlov MJ, Nale DP, Micev MT, Kovacevic PA and Stamenkovic AB: Validation and feasibility of ex vivo sentinel lymph node 'mapping' by methylene blue in colorectal cancer. Hepatogastroenterology 57: 1113-1118, 2010.

23. Park JS, Chang IT, Park SJ, Kim BG, Choi YS, Cha SJ, Park ES and Kwon GY: Comparison of ex vivo and in vivo injection of blue dye in sentinel lymph node mapping for colorectal cancer. World J Surg 3: 539-546, 2009.

24. Resch A and Langner C: Lymph node staging in colorectal cancer: Old controversies and recent advances. World J Gastroenterol 46 : 8515-8526, 2013

25. Frasson M, Faus C, Garcia-Granero A, Puga R, Flor-Lorente B, Cervantes A, Navarro S and Garcia-Granero E: Pathological evaluation of mesocolic resection quality and ex vivo methylene blue injection: What is the impact on lymph node harvest after colon resection for cancer? Dis Colon Rectum 2: 197-204, 2012.

26. Liu J, Huang P, Zheng Z, Chen T and Wei H: Modified methylene blue injection improves lymph node harvest in rectal cancer. ANZ J Surgery 4: 247-251, 2017.

27. Bembenek A, String A, Gretschel S and Schlag PM: Technique and clinical consequences of sentinel lymph node biopsy in colorectal cancer. Surg Oncol 17: 183-193, 2008

28. Balcangiu-Stroescu AE, Tănăsescu MD, Diaconescu AC, Răducu L, Bălan DG, Mihai A, Tănase M, Stănescu II and Ionescu D: Diabetic nephropathy: A concise assessment of the causes, risk factors and implications in diabetic patients. Rev Chim Buchar. 69:3118-3121.2018.
29. Timofte D, Mandita A, Balcangiu-Stroescu AE, Balan DG, Raducu L, Tanasescu MD, Diaconescu AC, Dragos D, Cosconel CI, Stoicescu SM, et al: Hyperuricemia and cardiovascular diseases- clinical and paraclinical correlations. Rev Chim Buchar 70: 1045-1046, 2019.

30. Tong GJ, Zhang GY, Liu J, Zheng ZZ, Chen Y, Niu PP and $\mathrm{Xu}$ XT: Comparison of the eighth version of the American joint committee on cancer manual to the seventh version for colorectal cancer: A retrospective review of our data. World J Clin Oncol 9: 148-161, 2018.

31. Di Berardino S, Capolupo GT, Caricato C and Caricato M: Sentinel lymph node mapping procedure in T1 colorectal cancer: A systematic review of published studies. Medicine (Baltimore) 98: e16310, 2019

32. Ong ML and Schofield JB: Assessment of lymph node involvement in colorectal cancer. World J Gastrointest Surg 8: 179-192, 2016.

33. Freitas AHA, Wainstein AJA and Nunes TA: Ex vivo sentinel lymph node investigation in colorectal cancer. J Coloproctol 33: 16-21, 2013.

34. Oh SY, Kim DY, Kim YB and Suh KW: Clinical application of sentinel lymph node mapping in colon cancer: In vivo vs. ex vivo techniques. Ann Surg Treat Res 87: 118-122, 2014.

This work is licensed under a Creative Commons Attribution-NonCommercial-NoDerivatives 4.0 International (CC BY-NC-ND 4.0) License. 